\title{
RESOLUTION TESTS OF CsI(Tl) SCINTILLATORS READ OUT BY PIN DIODES
}

\author{
W.G. Gong, Y.D. Kim, G. Poggi, Z. Chen, C.K. Gelbke, W.G. Lynch, \\ M.R. Maier, T. Murakami, M.B. Tsang, and H.M. Xu \\ National Superconducting Cyclotron Laboratory \\ Michigan State University, East Lansing, Michigan 48824 \\ K. Kwiatkowski \\ Department of Chemistry and Indiana University Cyclotron Facility \\ Indiana University, Bloomington, Indiana 47405
}

The study of intermediate energy heavy ion reactions often requires the detection of coincident light particles $(p, d, t, \alpha . .$.$) emitted with energies ranging from the exit chan-$ nel Coulomb barriers up to several hundred $\mathrm{MeV}$. For moderate resolution requirements, plastic scintillators are adequate. Better resolution can be obtained with inorganic scintillators. $\mathrm{CsI}(\mathrm{Tl})$ scintillators have a spectral response which is well matched to that of silicon photodiodes which exhibit excellent long term stability when read out with good quality charge sensitive preamplifiers. We measured the energy resolution for a cylindrical $\mathrm{CsI}(\mathrm{Tl})$ scintillator of $38 \mathrm{~mm}$ diameter and $100 \mathrm{~mm}$ length read out by a $400 \mathrm{~mm}^{2}$ PIN diode. For this purpose the IUCF low intensity $200 \mathrm{MeV}$ proton beam was degraded to $178 \mathrm{MeV}$ by passing it through an aluminum absorber and then collimating it with $50 \mathrm{~mm}$ thick $\mathrm{Cu}$ collimators with circular holes of 3 or $25 \mathrm{~mm}$ diameter, respectively. Energy resolutions of $1.4 \%$ and $1.7 \%$ were measured with the CsI detector for the collimators of 3 and $25 \mathrm{~mm}$ diameter, respectively. The resolution was found to be limited by local nonuniformities causing variations of the scintillation efficiency of several percent. ${ }^{1}$

As an illustration of these local fluctuations in the scintillation efficiency, Fig. 1 shows the position dependence of the scintillator response as measured with collimated ${ }^{137} \mathrm{Cs}$ $\gamma$-ray source. Scans of the detector response along two directions perpendicular to the cylinder axis (denoted by $\mathrm{X}$ and $\mathrm{Y}$ ) revealed non-trivial variations of the peak position as a function of the collimator position. The asymmetries in the detector response measured with collimated protons and the $\gamma$-rays exhibit similar qualitative trends. Of course there are quantitative differences since the two tests sample different (though partly overlapping) regions of the crystal.

More details of these scintillator tests can be found in Ref. 1 .

1. W.G. Gong, Y.D. Kim, G. Poggi, Z. Chen, C.K. Gelbke, W.G. Lunch, M.R. Maier, T. Murakami, M.B. Tsang, H.M. Xu, and K. Kwiatkowski, Nucl. Instr. and Meth. A268, 190 (1988). 


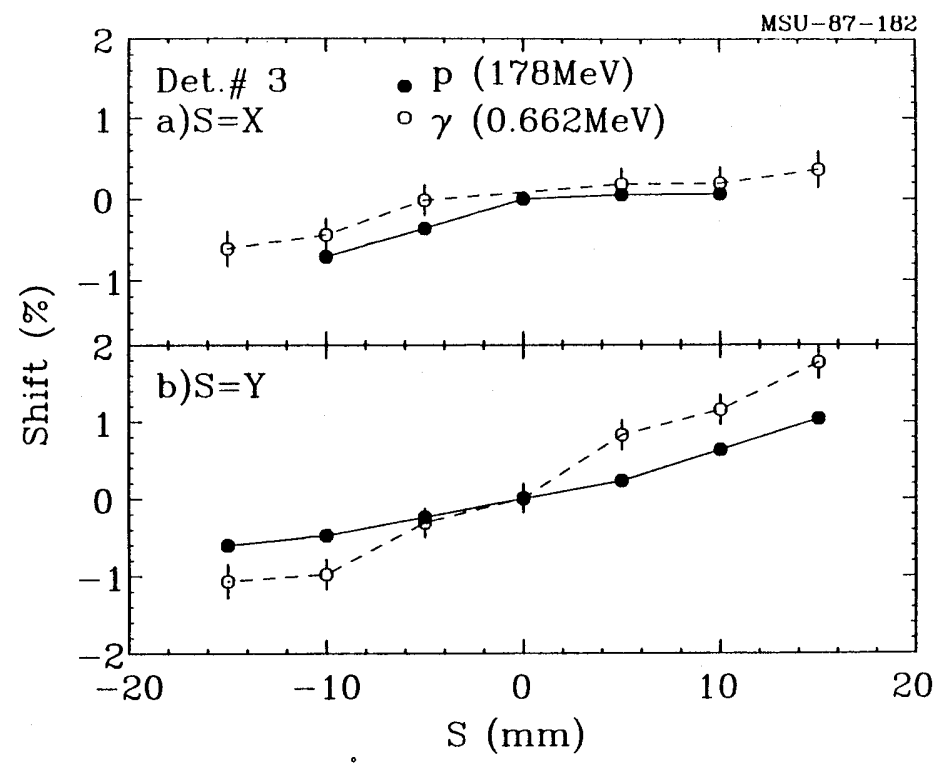

Figure 1. Position sensitive response of a $\mathrm{CsI}(\mathrm{Tl})$ crystal as detected by collimated protons of $178 \mathrm{MeV}$ (solid points) entering the front face $(\mathrm{Z}=0)$ of the crystal with the PIN diode mounted at the rear end $(\mathrm{Z}=112 \mathrm{~mm})$ and by collimated $\gamma$-rays (open points) entering the rear face $(\mathrm{Z}=100 \mathrm{~mm})$ of the crystal with the PIN diode mounted at the front end $(\mathrm{Z}=-12 \mathrm{~mm})$. Part a) shows measurements as a function of the $\mathrm{X}$-coordinate of the collimator keeping $\mathrm{Y}=0$; part $\mathrm{b}$ ) shows measurements as a function of the $\mathrm{Y}$-coordinate of the collimator keeping $\mathrm{X}=0$. 